\title{
The association between Toxoplasma gondii and type 2 diabetes mellitus: a systematic review and meta-analysis of human case- control studies
}

\author{
Aus Molan ${ }^{1 *} \mathbb{D}$, Kazunori Nosaka ${ }^{1}$, Michael Hunter ${ }^{2,3}$ and Wei Wang ${ }^{1,4}$
}

\begin{abstract}
Objectives: An emerging field of research is examining the association of infectious and environmental pathogens with type 2 diabetes mellitus (T2DM). An understudied pathogen of interest is the protozoan parasite Toxoplasma gondii (T. gondii). The objective of this study was to investigate the possible correlation between T. gondii infection and T2DM. We conducted a systematic review and meta-analysis on the currently available $T$. gondii seroprevalence data from case-control studies looking at subjects with T2DM in comparison to healthy controls to estimate the risk of T2DM.
\end{abstract}

Methods: Ten electronic databases were searched using specific Medical Subject Headings terms without language or date restrictions. Fixed and random effects models were used to determine odds ratios with statistical significance being set at 5.0\%.

Results: Ten publications reporting T. gondii seroprevalence from 4072 subjects met the eligibility criteria. Seven of these studies reported a significant association between T. gondii infection and T2DM $(p<0.05)$. The overall weighted prevalence of T. gondii infection in subjects with T2DM was 47.8\% (range 6.4-65.1\%) in comparison to $25.9 \%$ (range $3.2-59.0 \%$ ) of healthy controls $(p<0.001$ ). The common odds ratio, calculated using a random effects model, was 2.32 (95\% Cl 1.66-3.24, $p<0.001)$.

Conclusions: T. gondii infection should continue to be regarded as a possible contributing factor in T2DM disease development. Further studies that include inflammatory biomarker analysis are warranted to determine the specific role of this parasite in the pathogenesis of T2DM.

Keywords: Toxoplasma gondii, Diabetes mellitus, Type 2, Prevalence, Public health, Meta-analysis, Systematic review

\section{Background}

Type 2 diabetes mellitus accounts for roughly $90 \%$ of all diagnosed diabetes cases worldwide (American Diabetes Association 2010). The disease is characterised by reduced insulin production and an inability of body tissues to fully respond to insulin (insulin resistance) (American Diabetes Association 2010; Prandota 2013). This is caused by an ongoing deterioration of the insulin secretory capacity of pancreatic $\beta$-cells that does not

\footnotetext{
*Correspondence: amolan@our.ecu.edu.au; ausmolan@gmail.com ${ }^{1}$ School of Medical and Health Sciences, Edith Cowan University, Joondalup, Western Australia, Australia

Full list of author information is available at the end of the article
}

allow compensation for an increased peripheral insulin demand (Donath et al. 2005). Worldwide, diabetes has an estimated prevalence of $9 \%$ among adults aged 18 years and older, and the International Diabetes Federation estimates that 642 million people will be living with diabetes by 2040, up from 425 million in 2017 (International Diabetes Federation 2017). This is mainly due to the increase in the number of people with T2DM as a result of the growing aged population, reduction in physical activity (risk factor), bad dietary habits (risk factor), the obesity epidemic (risk factor), and the decreased mortality rates in diabetics (International Diabetes Federation 2017; World Health Organization 2014). The 
majority of individuals with T2DM are obese, and obesity itself has been reported to cause some degree of insulin resistance (American Diabetes Association 2010). In fact, the significant increase in the incidence and prevalence of diabetes over the last two decades can be largely explained by the global epidemic of obesity (Eckel et al. 2011; World Health Organization 2016). However, there may also be additional unidentified novel risk factors, such as subclinical inflammation caused by infectious agents, that contribute to this rising prevalence of T2DM (Pradhan 2007). In this regard, an emerging field of research is beginning to investigate the potential of infectious and environmental pathogens to cause lowgrade inflammation that may facilitate the risk and development of various metabolic conditions, including diabetes and obesity (Prandota 2013).

An understudied pathogen of potential interest in this field is the protozoan parasite Toxoplasma gondii ( $T$. gondii). This obligate intracellular parasite infects approximately one third of the world's population and is considered one of the most successful human parasites (Centers for Disease Control and Prevention 2015; Halonen and Weiss 2013). In fact, the Centers for Disease Control (CDC) has considered T. gondii as one of the top "Five Neglected Parasitic Infections" due to the severity of illness, high incidence, and potential for prevention (Centers for Disease Control and Prevention 2017). Humans acquire T. gondii infection by the ingestion of food, water, or soil contaminated by oocysts from the definitive hosts, cats. Even though T. gondii is distributed worldwide and is considered to have the widest host range of any parasite, only one species (gondii) exists in the genus Toxoplasma, with cats being the only known definitive host in which sexual development takes place (Dupey 2008).

While several studies have demonstrated a positive correlation between $T$. gondii infection and numerous neurological disorders and cancers (Prandota 2013; Cong et al. 2015; Henriques et al. 2009), T. gondii infection in individuals with T2DM has received little recognition. However, this area has recently gained rapid attention with the majority of available data being produced from studies conducted within the last few years. The objective of the current study was to estimate the risk of T2DM due to $T$. gondii infection by conducting a systematic review and meta-analysis of published studies examining the seroprevalence rates of $T$. gondii in subjects with T2DM in comparison to those without T2DM.

\section{Materials and methods}

\section{Strategy for literature search}

To identify relevant published studies on the possible relationship between T. gondii infection and T2DM, our group conducted a systematic literature review and meta- analysis on published literature with no language or date restrictions (from inception until February 2019) from 10 electronic databases (Google Scholar, Science Direct, Embase, PubMed, PLOS ONE, Web of Knowledge, SciELO, MyAIS, Free Medical Journals, and Scopus). The analysis was conducted according to the recommendations of the PRISMA Statement (Moher et al. 2009). The Medical Subject Headings terms used in the search were "Toxoplasma" OR "Toxoplasma gondii" OR "toxoplasmosis" OR "T. gondii" OR "TORCH" [Toxoplasmosis, Other (syphilis, varicella-zoster, parvovirus B19), Rubella, Cytomegalovirus (CMV), and Herpes infections] combined with (AND) "type 2 diabetes mellitus" OR "type 2 diabetes" OR "T2DM" OR "diabetes".

\section{Selection of studies}

Potentially relevant articles were initially selected based on title content followed by abstract content. The retained articles were read in full and screened for eligibility using a checklist of inclusion-exclusion criteria. All selected studies had to meet the following inclusion criteria: (i) cross-sectional observational studies using a case-control design, (ii) T2DM must be the disease state and $T$. gondii infection as the exposure, (iii) cases must be subjects diagnosed with T2DM, (iv) participants of the control group must be subjects without T2DM selected from the normal population, (v) sample sizes must be suitably estimated, and (vi) diagnosis of $T$. gondii infection must be based on the following standard laboratory detection methods: serological examination of $T$. gondii IgG and/or IgM antibodies, indirect fluorescent antibody test (IFAT), immunohistochemical (IHC) staining, or molecular methods detecting $T$. gondii DNA; where positive results were characterised by the presence of IgG and/or IgM or a positive IFAT test or a positive IHC stain or the detection of T. gondii DNA; and negative results were defined as a lack of IgG or IgM antibodies, a negative IFAT test, a negative IHC stain, or no detection of T. gondii DNA. Likewise, studies were excluded if they were (i) animal studies, (ii) repeated studies, (iii) abstracts, and (iv) studies which only included T2DM subjects without a control group. Any discrepancies with the final selection of studies were resolved by discussion and consensus with another author.

\section{Data collection and analysis}

The following information was extracted from each study: title, primary author, year of publication, location of the study, aims, methods, results, characteristics of the study population including collection criteria, numbers of case and control subjects, and diagnostic methods used in the diagnosis of T2DM and detection of $T$. gondii infection. Additionally, we also examined the reference lists of full-text publications and text books 
to identify any additional studies not retrieved by the initial database search.

The meta-analysis was performed using MedCalc (MedCalc Software, Korea) version 18.11.3. The common odds ratios (OR) and corresponding 95\% confidence intervals $(\mathrm{CI})$ were calculated for each individual study, and used as the measure of association between T2DM and T. gondii infection. Likewise, the ORs and respective 95\% CIs were calculated for the overall (pooled) estimates employing both random and fixed effect models. Consequently, a forest plot summarising the study statistics including the ORs and relative weights (\%, for both fixed and random effect models) was generated. The implication of the pooled ORs was determined by the $Z$ test. Heterogeneity among the studies was examined using the Cochrane $\mathrm{Q}$ and $\mathrm{I}^{2}$ tests. Publication bias was assessed using the Egger's regression test that is illustrated by a funnel plot of standard error by log odds ratio of the selected studies using the random effect model. MedCalc uses the Mantel-Haenszel method (Mantel and Haenszel 1959) for calculating the weighted pooled odds ratio under the fixed effects model. The heterogeneity statistic is incorporated to calculate the summary odds ratio under the random effects model (DerSimonian and Laird 1986).

\section{Results}

The results of the literature search are presented in Fig. 1. From the 10 databases searched (Google Scholar, Science Direct, Embase, PubMed, PLOS ONE, Web of Knowledge, SciELO, MyAIS, Free Medical Journals, and Scopus), a total of 1763 published papers were eligible under the predefined search terms. From these, 1440 were cited more than once. Of the remaining 323 papers, 298 were eliminated as they were not epidemiological studies. The remaining 25 papers were read as abstracts with 12 being rejected due to being literature reviews or animal studies. The residual 13 papers ( $\mathrm{Li}$ et al. 2018; Khalili et al. 2018; Han et al. 2018; Molan and Ismail 2017; AlvaradoEsquivela et al. 2017; Saheb 2017; Nihad and Hamad 2017; Hemida et al. 2017; Saki and Shafieenia 2016; Modrek et al. 2015a; Siyadatpanah et al. 2013; Shirbazou et al. 2013; Gokce et al. 2008), as summarised in Table 1, were read in full and three studies (Han et al. 2018; Hemida et al. 2017; Modrek et al. 2015a) were excluded as they lacked a control group and hence did not meet the inclusion criteria. The references of the review articles did not add any new studies. Consequently, ten studies were retained for the final analysis ( $\mathrm{Li}$ et al. 2018; Khalili et al. 2018; Molan and Ismail 2017; Alvarado-Esquivela et al. 2017; Saheb 2017; Nihad and Hamad 2017; Saki and Shafieenia 2016; Siyadatpanah et al. 2013; Shirbazou et al. 2013; Gokce et al. 2008) (Table 2).
The majority of the studies were conducted in the last 5 years with a significant output of eight studies from the Middle East (Iran, $n=4$; Iraq, $n=3$; and Turkey, $n$ $=1$ ), followed by China and Brazil with one study each. Six studies (Li et al. 2018; Khalili et al. 2018; Molan and Ismail 2017; Alvarado-Esquivela et al. 2017; Saheb 2017; Gokce et al. 2008) defined their diabetic case subjects according to the type of diabetes while the remaining four studies used the term "diabetic" (Table 1). Four studies (Li et al. 2018; Nihad and Hamad 2017; Saki and Shafieenia 2016; Siyadatpanah et al. 2013) recruited cases and control subjects from hospitals while another four studies (Khalili et al. 2018; Molan and Ismail 2017; Alvarado-Esquivela et al. 2017; Saheb 2017) recruited their cases and control subjects from clinics. The two residual studies did not specify the source of recruitment of the cases and controls. With regard to case-control matching for age and gender, only six studies ( $\mathrm{Li}$ et al. 2018; Khalili et al. 2018; Alvarado-Esquivela et al. 2017; Siyadatpanah et al. 2013; Shirbazou et al. 2013; Gokce et al. 2008) performed this. All studies employed commercially available laboratory IgG techniques to determine T. gondii infection status of their case and control subjects. In addition to the IgG technique, some studies also utilised the following additional diagnostic methods to further aid in the diagnosis of $T$. gondii infection status, as is often recommended (Pappas et al. 2009): commercial ELISA IgM (Li et al. 2018; Molan and Ismail 2017; Nihad and Hamad 2017; Saki and Shafieenia 2016; Siyadatpanah et al. 2013; Gokce et al. 2008), IgM CLIA (Khalili et al. 2018), IgM IFAT (Saki and Shafieenia 2016; Gokce et al. 2008), and IgM ELFA assays (Alvarado-Esquivela et al. 2017).

Table 2 summarizes the main findings of each individual study in addition to the ORs, respective 95\% CIs, and $p$ values. Only one study (Gokce et al. 2008) recruited subjects from both adults and adolescents. The total number of subjects in all included studies was 4072, of whom $60.6 \%$ (2467) had diabetes. The seroprevalence of $T$. gondii infection in diabetic patients ranged from 6.4 to $65.1 \%$ in comparison to 3.2 to $59.0 \%$ for healthy controls. The overall weighted prevalence of T. gondii infection in diabetic subjects was $47.8 \%(n=1$, $180)$ in comparison to $25.9 \%(n=416)$ in healthy controls. This difference was significant $(p<0.001)$. The seroprevalence of $T$. gondii is therefore higher in subjects with T2DM than health controls. Hence, all except three studies (Khalili et al. 2018; Alvarado-Esquivela et al. 2017; Siyadatpanah et al. 2013) reported a significant relationship between $T$. gondii infection and T2DM $(p<0.05)$. The pooled ORs of all included studies ranged from 1.08 (95\% CI $0.69-1.70)$ to 4.52 (95\% CI 3.26-6.28) with six of these results being significant $(p<$ $0.05)$. A forest plot of the individual study and pooled 


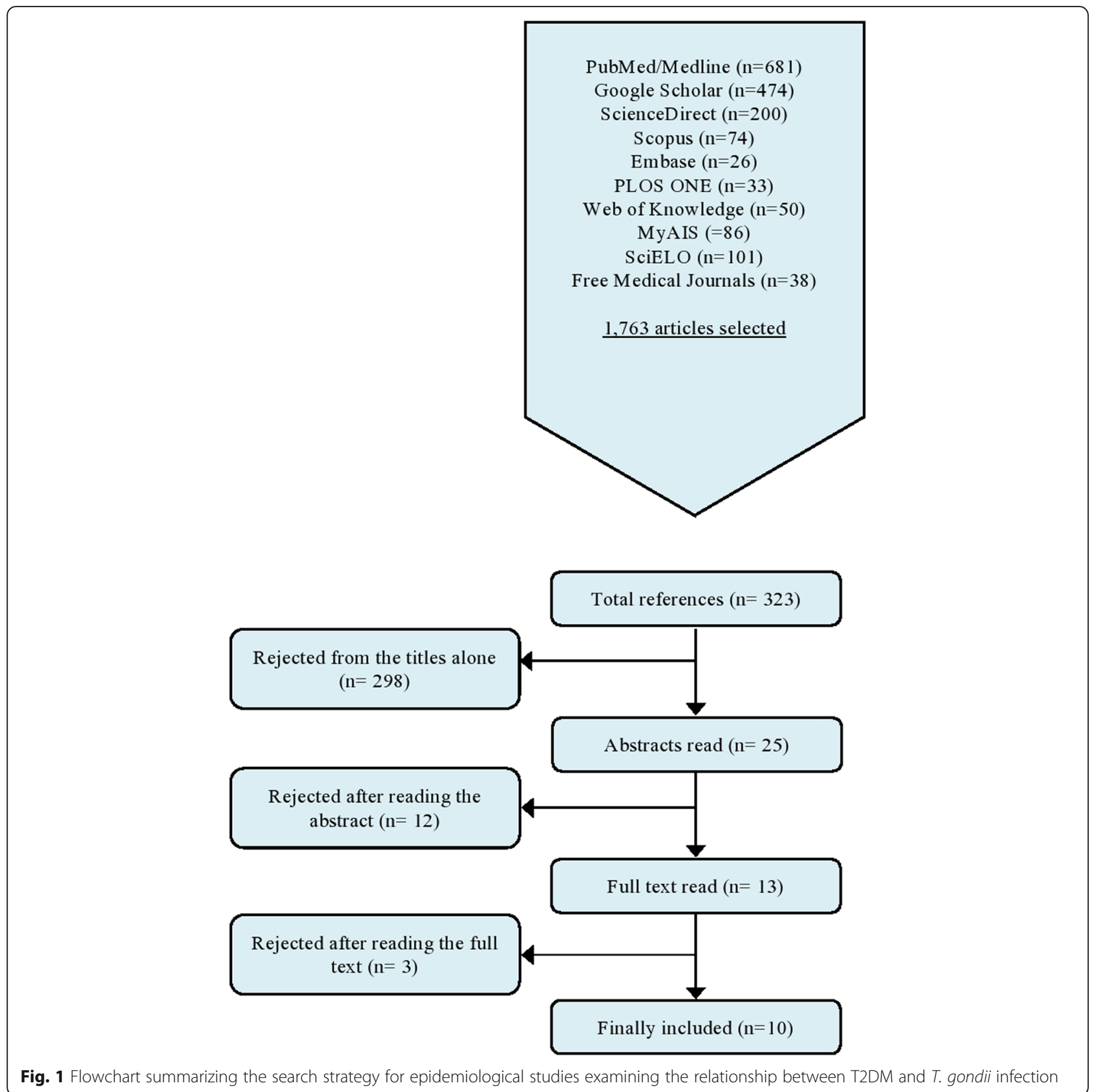

ORs is presented in Fig. 2. The common fixed effects model OR was calculated at 2.57 (95\% CI 2.22-2.99, $p<$ $0.001)$. However, due to the significant heterogeneity $(Q$ $=38.566, p<0.0001, I^{2}=76.66 \%$, Table 3 ), the common OR was estimated using a random effects model (2.32, 95\% CI 1.66-3.24, $p<0.001$ ). Accordingly, subjects with T. gondii infection exposure have a 2.3-fold higher risk of having diabetes when compared to unexposed subjects. Publication bias was not evident among the included studies, as displayed in the relative symmetry of the funnel plot (Fig. 3). However, due to the relatively low number of included studies, further tests for funnel plot asymmetry were not conducted as the test power will be too low to differentiate real asymmetry from coincidence.

\section{Discussion}

Our group performed a systematic review and metaanalysis of the available literature to evaluate the relationship between $T$. gondii infection and T2DM. Ten different databases were searched without date and language restrictions making the literature search comprehensive with minimal publication bias. However, it is possible that our search may have missed studies available exclusively in 
Table 1 Description of studies identified as relevant in the systematic literature search looking for an association between T. gondii and diabetes mellitus

\begin{tabular}{|c|c|c|c|c|c|c|c|c|c|}
\hline Reference & $\begin{array}{l}\text { Year } \\
\text { published }\end{array}$ & Country & Diabetes & $\begin{array}{l}\text { Study } \\
\text { type }\end{array}$ & Source 1 & Definition & Source 2 & Matching* & Measure of exposure \\
\hline Li et al. (Li et al. 2018) & 2018 & China & $\begin{array}{l}\mathrm{T} 1+ \\
2 \mathrm{DM}\end{array}$ & $\mathrm{CC}$ & Hospital & Unspecified & Hospital & Yes & $\begin{array}{l}\text { IgG/IgM ELISA (Demeditec } \\
\text { Diagnostics, Germany) }\end{array}$ \\
\hline $\begin{array}{l}\text { Khalili et al. (Khalili et al. } \\
\text { 2018) }\end{array}$ & 2018 & Iran & $\begin{array}{l}\mathrm{T} 1+ \\
2 \mathrm{DM}\end{array}$ & $\mathrm{CC}$ & Clinics & Unspecified & Clinics & Yes & $\begin{array}{l}\text { IgG/lgM CLIA (Disorin, } \\
\text { Italy) }\end{array}$ \\
\hline Han et al. (Han et al. 2018) & 2018 & China & $\mathrm{T} 2 \mathrm{DM}$ & Case & Hospital & Yes & N/A & N/A & $\begin{array}{l}\text { IgG ELISA (Haitai Biological } \\
\text { Pharmaceuticals, China) }\end{array}$ \\
\hline $\begin{array}{l}\text { Molan \& Ismail (Molan and } \\
\text { Ismail 2017) }\end{array}$ & 2017 & Iraq & $\begin{array}{l}\mathrm{T} 1+ \\
2 \mathrm{DM}\end{array}$ & $\mathrm{CC}$ & Clinics & Unspecified & Clinics & No & $\begin{array}{l}\text { IgG/IgM ELISA (ACON } \\
\text { Labs, USA) }\end{array}$ \\
\hline $\begin{array}{l}\text { Alvarado-Esquivel et al. } \\
\text { (Alvarado-Esquivela et al. } \\
\text { 2017) }\end{array}$ & 2017 & Brazil & $\begin{array}{l}\mathrm{T} 1+ \\
2 \mathrm{DM}\end{array}$ & $\mathrm{CC}$ & Clinics & Unspecified & Clinics & Yes & $\begin{array}{l}\text { IgG ELIFA \& VIDAS ELFA } \\
\text { IgM (BioMerieux, France) }\end{array}$ \\
\hline Saheb (Saheb 2017) & 2017 & Iraq & $\mathrm{T} 2 \mathrm{DM}$ & $\mathrm{CC}$ & Clinics & Unspecified & Clinics & No & $\begin{array}{l}\text { IgG ELISA (BioCheck Inc., } \\
\text { USA) }\end{array}$ \\
\hline $\begin{array}{l}\text { Nihad \& Hamad (Nihad and } \\
\text { Hamad 2017) }\end{array}$ & 2017 & Iraq & Diabetic & $\mathrm{CC}$ & Hospital & Unspecified & Hospital & No & $\begin{array}{l}\text { lgG/lgM ELISA (BioCheck } \\
\text { Inc., USA) }\end{array}$ \\
\hline $\begin{array}{l}\text { Hemida et al. (Hemida et al. } \\
\text { 2017) }\end{array}$ & 2017 & Egypt & $\begin{array}{l}\mathrm{T} 1+ \\
2 \mathrm{DM}\end{array}$ & Case & Hospital & Unspecified & N/A & N/A & $\begin{array}{l}\text { IgG/IgM ELISA (Calbiotech } \\
\text { Inc., USA) }\end{array}$ \\
\hline $\begin{array}{l}\text { Saki et al. (Saki and } \\
\text { Shafieenia 2016) }\end{array}$ & 2016 & Iran & Diabetic & $\mathrm{CC}$ & Hospital & Unspecified & Hospital & No & $\begin{array}{l}\text { IgG/IgM ELISA (Trinity } \\
\text { Biotech, USA) \& IFAT kit }\end{array}$ \\
\hline $\begin{array}{l}\text { Modrek et al. (Modrek et al. } \\
\text { 2015a) }\end{array}$ & 2015 & Iran & Diabetic & Case & Hospital & Unspecified & N/A & N/A & $\begin{array}{l}\text { IgG/IgM (DRG instrument } \\
\text { GmbH, Germany) }\end{array}$ \\
\hline $\begin{array}{l}\text { Siyadatpanah et al. } \\
\text { (Siyadatpanah et al. 2013) }\end{array}$ & 2013 & Iran & Diabetic & $C C$ & Hospital & Unspecified & Hospital & Yes & $\begin{array}{l}\text { IgG/IgM ELISA (VIRO, } \\
\text { Germany) }\end{array}$ \\
\hline $\begin{array}{l}\text { Shirbazou et al. (Shirbazou } \\
\text { et al. 2013) }\end{array}$ & 2013 & Iran & Diabetic & $C C$ & Unspecified & Unspecified & Unspecified & Yes & $\begin{array}{l}\text { IgG ELISA (Pishtaz Teb } \\
\text { Zaman Diagnostics, Iran) }\end{array}$ \\
\hline $\begin{array}{l}\text { Gokce et al. (Gokce et al. } \\
\text { 2008) }\end{array}$ & 2008 & Turkey & $\mathrm{T} 2 \mathrm{DM}$ & $\mathrm{CC}$ & Unspecified & ADA & Unspecified & Yes & $\begin{array}{l}\operatorname{lgG} / \operatorname{lgM} \text { ELISA and IFAT } \\
\text { (Euroimmun) }\end{array}$ \\
\hline
\end{tabular}

*Matching is by age and gender alone; source 1, source of diabetes mellitus cases; source 2, source of non-type 2 diabetes mellitus controls; definition, definition of diabetes mellitus cases. Highlight signifies the studies retained for the meta-analysis (Table 2)

CC case-control, IgG immunoglobulin G, IgM immunoglobulin M, ELISA enzyme-linked immunosorbent assay, CLIA chemiluminescence immunoassay, ELIFA enzyme-linked immunofiltration assay, ELFA enzyme-linked fluorescent assay, IFAT immunofluorescence antibody test

Table 2 Description of data extracted from the retained studies included in the meta-analysis searching for an association between T. gondii and diabetes mellitus

\begin{tabular}{|c|c|c|c|c|c|c|c|c|c|c|}
\hline \multirow[t]{2}{*}{ Reference } & \multirow[t]{2}{*}{$n$} & \multirow[t]{2}{*}{ Age } & \multirow{2}{*}{$\begin{array}{l}\mathrm{DM}+ \\
(n)\end{array}$} & \multirow{2}{*}{$\begin{array}{l}\text { CTRL } \\
-(n)\end{array}$} & \multirow{2}{*}{$\begin{array}{l}\text { DM \& TG+, } \\
n(\%)\end{array}$} & \multirow{2}{*}{$\begin{array}{l}\text { CTRL \& } \\
\text { TG+, } n \text { (\%) }\end{array}$} & \multirow[t]{2}{*}{ OR $(95 \% \mathrm{Cl})$} & \multirow[t]{2}{*}{$p$ value } & \multicolumn{2}{|c|}{ Weight (\%) } \\
\hline & & & & & & & & & Fixed & Random \\
\hline Li et al. (Li et al. 2018) & 800 & Adults & 400 & 400 & $94(23.5 \%)$ & $47(11.8 \%)$ & $2.31(1.57-3.38)$ & $<0.001$ & 15.82 & 11.95 \\
\hline Khalili et al. (Khalili et al. 2018) & 200 & Adults & 100 & 100 & $63(63.0 \%)$ & $59(59.0 \%)$ & $1.18(0.67-2.09)$ & 0.562 & 7.14 & 10.05 \\
\hline Molan \& Ismail (Molan and Ismail 2017) & 584 & Adults & 381 & 203 & $248(65.1 \%)$ & $68(33.4 \%)$ & $3.70(2.58-5.30)$ & $<0.001$ & 17.87 & 12.17 \\
\hline Alvarado-Esquivel et al. & 312 & Adults & 156 & 156 & $10(6.4 \%)$ & $5(3.2 \%)$ & $2.07(0.69-6.20)$ & 0.18 & 1.92 & 5.62 \\
\hline Saheb (Saheb 2017) & 270 & Adults & 172 & 98 & $96(55.8 \%)$ & $38(38.8 \%)$ & $1.99(1.20-3.31)$ & 0.007 & 9.04 & 10.70 \\
\hline Nihad \& Hamad (Nihad and Hamad 2017) & 145 & Adults & 100 & 45 & $31(31.0 \%)$ & $5(11.1 \%)$ & $3.59(1.29-9.99)$ & 0.010 & 2.21 & 6.11 \\
\hline Saki et al. (Saki and Shafieenia 2016) & 220 & Adults & 110 & 110 & $47(42.7 \%)$ & $24(21.8 \%)$ & $2.67(1.48-4.82)$ & 0.001 & 6.65 & 9.84 \\
\hline Siyadatpanah et al. (Siyadatpanah et al. 2013) & 300 & Adults & 150 & 150 & $79(52.7 \%)$ & $76(50.6 \%)$ & $1.08(0.69-1.70)$ & 0.729 & 11.26 & 11.24 \\
\hline Shirbazou et al. (Shirbazou et al. 2013) & 184 & Adults & 91 & 93 & $55(60.4 \%)$ & $38(40.9 \%)$ & $2.21(1.23-3.99)$ & 0.008 & 6.65 & 9.84 \\
\hline Gokce et al. (Gokce et al. 2008) & 1057 & $15-88$ & 807 & 250 & $457(56.6 \%)$ & $56(22.4 \%)$ & $4.52(3.26-6.28)$ & $<0.001$ & 21.44 & 12.46 \\
\hline Total (fixed) & 4072 & - & 2467 & 1605 & $1180(47.8 \%)$ & $416(25.9 \%)$ & $2.57(2.22-2.99)$ & $<0.001$ & $100 \%$ & $100 \%$ \\
\hline Total (random) & 4072 & - & 2467 & 1605 & $1180(47.8 \%)$ & $416(25.9 \%)$ & $2.32(1.66-3.24)$ & $<0.001$ & $100 \%$ & $100 \%$ \\
\hline
\end{tabular}




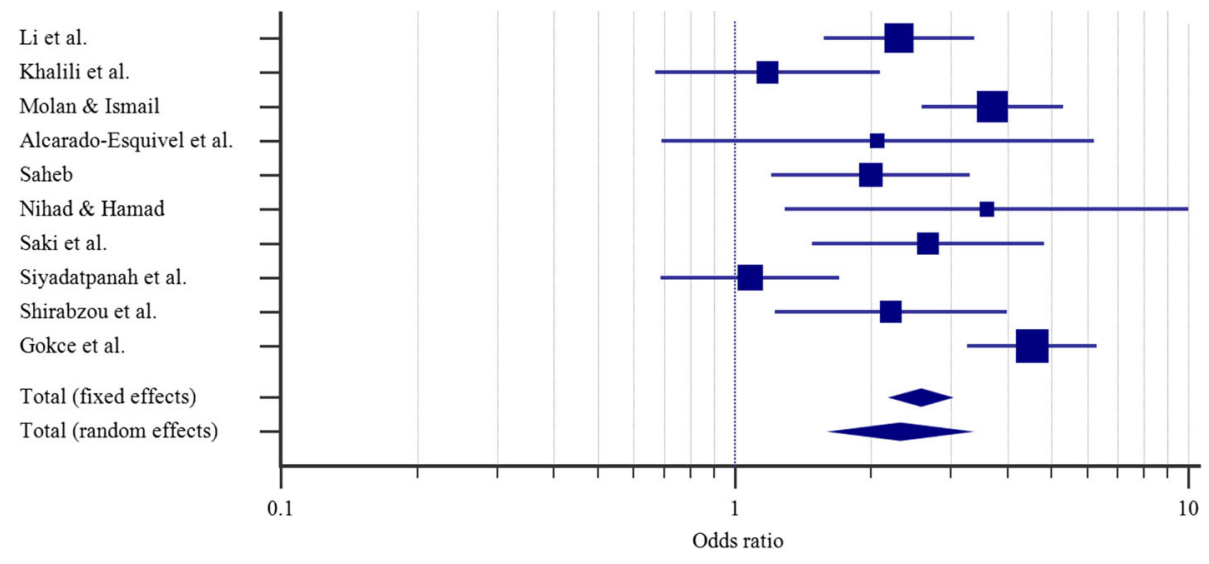

Fig. 2 Forest plot of odds ratios for individual studies describing epidemiological correlations between T. gondii infection and diabetes mellitus

other languages. One of the most striking findings to come out of this meta-analysis is that little human data exists on the relationship between $T$. gondii and T2DM despite the fact that $T$. gondii infects approximately one third of the world's population (Centers for Disease Control and Prevention 2015; Halonen and Weiss 2013). Seven (Li et al. 2018; Molan and Ismail 2017; Saheb 2017; Nihad and Hamad 2017; Saki and Shafieenia 2016; Shirbazou et al. 2013; Gokce et al. 2008) of the 10 studies found that subjects with T2DM had significantly higher $(p<0.05)$ prevalence rates of $T$. gondii when compared to healthy controls, therefore concluding that a significant relationship exists between $T$. gondii infection and T2DM. The remaining three studies (Khalili et al. 2018; Alvarado-Esquivela et al. 2017; Siyadatpanah et al. 2013) did not find a statistically significant difference in prevalence of the parasite in diabetic and non-diabetic individuals. The degree of risk in the present analysis, estimated using the random effect model (OR, 2.32; 95\% CI 1.66-3.24; $p<0.001$ ), matches previously published meta-analyses conducted by Majidiani et al. (OR, 2.39; 95\% CI 1.20-4.75; $p=0.013$ ) (Majidiani et al. 2016) and our group (OR, 2.34; 95\% CI 1.17-4.64; $p=0.016$ ) (Molan et al. 2018). While our inclusion/exclusion criteria were more stringent than the Majidiani et al. meta-analysis, both previous reviews only included the four available studies at that time (Saki and Shafieenia 2016; Siyadatpanah et al. 2013; Shirbazou et al. 2013; Gokce et al. 2008). Our results therefore demonstrate that $T$. gondii infection is associated with a 2.3-fold increased risk of T2DM in comparison to unexposed subjects. This warrants further research to determine whether $T$. gondii increases the risk of developing T2DM and possible mechanisms underlying this association.

None of the studies found during our search were from Europe, USA, Asia Pacific, and Africa. In addition, most of Asia, the Middle East, and South America also remains unaddressed albeit four papers from Iran, three from Iraq, one from Turkey, one from China, and one from Brazil. The majority of the studies selected for analysis in the present study had moderate adult sample sizes with the exception of Gokce et al. (Gokce et al. 2008), Li et al (Li et al. 2018), and Molan et al. (Molan and Ismail 2017) who had 1057 age- and gender-matched subjects aged 18-55 years, 800 age- and gender-matched adults, and 584 unmatched adults, respectively. All studies shared common fundamental limitations which also may account for the large observed heterogeneity in the present study. Firstly, there is a lack of standardisation with regard to the criteria used to define various parameters, especially the diagnosis of T2DM (methodical heterogeneity). This includes defining inclusion and exclusion criteria consistent with the latest definitions of diabetes by the WHO. Secondly, there is no mention of having excluded individuals with psychiatric conditions, including personality disorders, from these studies (clinical heterogeneity). This feature is important because an association between positive T. gondii serology and various forms of serious mental illness has been previously reported (Halonen and Weiss 2013; Del Grande et al. 2017). Thirdly, the studies were

Table 3 Calculated data extracted from the included studies to assess heterogeneity in the meta-analysis searching for an association between T. gondii and diabetes mellitus using both fixed and random effect models

\begin{tabular}{|c|c|c|c|c|c|c|c|c|c|c|}
\hline \multirow[t]{2}{*}{ Model } & \multicolumn{4}{|c|}{ Effect size and 95\% interval } & \multicolumn{2}{|c|}{ Test of null (two-tail) } & \multicolumn{4}{|c|}{ Heterogeneity } \\
\hline & Number of studies & Point estimate & Lower limit & Upper limit & $Z$ value & $p$ value & Q value & $\mathrm{df}(\mathrm{Q})$ & $p$ value & $P^{2}(\%, 95 \% \mathrm{Cl})$ \\
\hline Fixed & 10 & 2.57 & 2.22 & 2.99 & 12.40 & $<0.001$ & 38.566 & 9 & $<0.0001$ & $76.66(56.96-87.35)$ \\
\hline Random & 10 & 2.32 & 1.66 & 3.24 & 4.92 & $<0.001$ & - & - & - & - \\
\hline
\end{tabular}




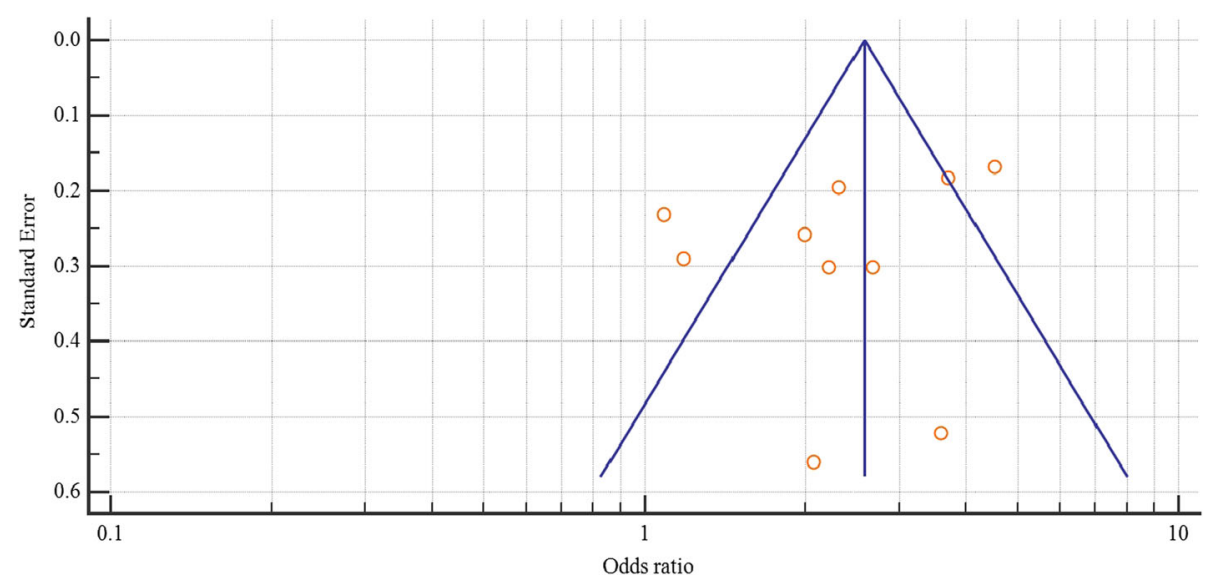

Fig. 3 Funnel plot of standard error by log odds ratio of the selected studies using the random effects model estimated by the Egger's regression test

not comparable in their methods of measuring $T$. gondii exposure, a fundamental factor that also increases methodical heterogeneity.

The role of $T$. gondii in the pathogenesis of T2DM remains unknown. One possible mechanism could be the inflammatory-mediated destruction of pancreatic $\beta$ cells which leads to the reduction in $\beta$ cell mass that ultimately contributes to the failure of the $\beta$ cell to produce enough insulin. This in turn would increase the risk of developing acute and chronic pancreatitis as well as diabetes (Donath et al. 2005; Butler et al. 2013). Additionally, it is known that T2DM is linked with an increased risk of developing acute pancreatitis (Gonzalez-Perez et al. 2010) and that $T$. gondii infection can cause pancreatic tissue necrosis (Waree 2008). Accordingly, individuals infected with $T$. gondii may be at increased risk of developing diabetes than uninfected individuals. Certainly, insulin has been shown to have a stimulatory effect on the in vitro replication of T. gondii (Oz 2014). Moreover, insulin and D-glucose have been shown to have a synergistic doseresponsive stimulating effect on the replication of $T$. gondii tachyzoites in vitro (Hassanain et al. 2014). Animal studies have also shown positive correlations between both the $T$. gondii parasite load and IgM antibody titre, and blood glucose levels in diabetic rats infected with $T$. gondii monitored at 15 -day intervals for $60 / 105$ days' post diabetes/T. gondii infection (Modrek et al. 2015b). During this period, Modrek et al. (Modrek et al. 2015b) observed a significant increase in blood glucose levels in $T$. gondii infected diabetic group when compared to the uninfecteddiabetic group. In addition, the elevated brain parasite load and IgM titre corresponded to the elevated glucose levels in infected diabetic group which led to the view that $T$. gondii infection can increase the risk of developing diabetes (Modrek et al. 2015b).
Furthermore, T. gondii infection may be associated with obesity due to the ability of the organism to alter inflammatory fat distribution as it resides in fatty tissues (Carter 2013). Obesity has been previously referred to as "a state of chronic inflammation" (Carter 2013), and it is therefore plausible that $T$. gondii could be involved in the obesity-T2DM paradigm. Excessive gestational weight gain was reported from two experiments conducted during pregnancy in $T$. gondii-infected woman compared with unaffected pregnant woman (Kankova et al. 2010; Flegr 2013). In addition, Reeves et al. (Reeves et al. 2013) reported a positive association between $T$. gondii seropositivity and obesity from a sample of 999 human adults. Their results showed that individuals with a positive $T$. gondii serology had twice the odds of being obese $\left(\mathrm{BMI}>30 \mathrm{~kg} / \mathrm{m}^{2}\right)$ when compared to seronegative individuals $(p=0.01)$. Infection with $T$. gondii may also act as a stimulus for motivational- and reward-driven behaviours (over-eating for example) via altered dopamine pathways. Animal models have shown that both dopamine release and availability of the rate-limiting enzyme in dopamine synthesis are influenced by $T$. gondii and that dopamine antagonists can block behaviour changes in rats infected with T. gondii (Prandovszky et al. 2011). Thus, the behavioural changes in the host which may be induced by $T$. gondii, may be driven by survival needs which ultimately could have inadvertent effects on eating patterns that promote obesity (Reeves et al. 2013). Accordingly, the association between T. gondii infection, T2DM, and obesity is very significant and could alter the current views on T2DM management.

Lastly, it has been argued that the simple explanation for the relationship between T. gondii and T2DM may be that diabetic patients have an increased susceptibility to parasitic infections due the possibility of a suppressed 
immune system, decreased arterial perfusion, and neuropathy (Bessman and Sapico 1992; Nazligulm et al. 2001). However, there is evidence to suggest otherwise. A study looking at parasitic infections in 200 diabetic (type $1, n=16$; type $2, n=184$ ) and 1024 nondiabetic individuals did not find a significant difference in the incidence of intestinal parasites between the T2DM and control groups (47\% and 56\%, respectively) (Nazligulm et al. 2001).

If the serological and inflammatory associations between T. gondii and T2DM are further replicated, it will warrant the need for further research to clarify whether $T$. gondii increases the risk of developing T2DM, and mechanisms that drive this relationship, if any. Current reports are limited to association studies and could not determine if there is a causal relationship between $T$. gondii and T2DM or obesity, including possible reverse causality (i.e. T2DM prompting the risk of $T$. gondii infection) or shared causality (i.e. common factor causing both T2DM and T. gondii infection). In addition, studies measuring only $T$. gondii IgG and IgM seropositivity cannot shed any light on the possible inflammatory association between T2DM and $T$. gondii infection. Therefore, future studies specifically designed to investigate the role of $T$. gondii in T2DM using inflammation as the main outcome are urgently needed in order to provide a more robust link between $T$. gondii infection and T2DM. To this extent, studies examining selected inflammatory biomarkers that are altered in the serum during, and in the absence of chronic T. gondii infection in subjects with T2DM vs. non-T2DM healthy controls will be highly valuable.

\section{Conclusions}

In light of the above reports, there is laboratory evidence to support the notion of an association between $T$. gondii infection and T2DM. If T. gondii is found to be involved in the inflammation process leading to T2DM, routine screening for $T$. gondii infection in individuals with T2DM would add to the risk prediction for diabetes to target individuals for early aggressive intervention. In future, focusing on T. gondii and reducing its risk factors could be used as an Achilles heel to reduce inflammation leading to a reduction in insulin resistance and an improvement in glucose tolerance in diabetic individuals.

\footnotetext{
Abbreviations

95\% Cl: 95\% Confidence interval; BMl: Body mass index; CDC: Centers for Disease Control; CLIA: Chemiluminescence immunoassay; ELFA: Enzymelinked fluorescent assay; ELISA: Enzyme-linked immunosorbent assay; IFAT: Indirect fluorescent antibody test; IgG: Immunoglobulin g; IgM: Immunoglobulin M; IHC: Immunohistochemical; T. gondii: Toxoplasma gondii; T2DM: Type 2 Diabetes Mellitus; WHO: World Health Organization
}

\section{Acknowledgements}

We thank the School of Medical and Health Sciences at Edith Cowan University, Joondalup, Western Australia.

\section{Authors' contributions}

All authors contributed to the conception and design of the study. AM, KN, and WW obtained ethical approval and acquired the data. AM performed the statistical analysis. $\mathrm{AM}$ and $\mathrm{MH}$ revised the draft manuscript for important intellectual content. All authors reviewed and edited drafts and approved the final manuscript for publication. All authors agree to be accountable for all aspects of the work in ensuring that questions related to the accuracy or integrity of any part of the work are appropriately investigated and resolved.

\section{Funding}

This work was supported by the Joint Project of the Australian National Health and Medical Research Council (NHMRC) and the National Natural Science Foundation of China (NSFC) (NHMRC APP1112767-NSFC 81561128020)

\section{Availability of data and materials}

All data generated or analysed during this study are included in this published article.

\section{Ethics approval and consent to participate}

Not applicable.

\section{Consent for publication}

Not applicable.

\section{Competing interests}

The authors declare that they have no competing interests.

\section{Author details}

${ }^{1}$ School of Medical and Health Sciences, Edith Cowan University, Joondalup, Western Australia, Australia. ${ }^{2}$ Busselton Population Medical Research Institute, Busselton, Western Australia, Australia. ${ }^{3}$ School of Population and Global Health, University of Western Australia, Nedlands, Western Australia, Australia. ${ }^{4}$ Key Municipal Laboratory of Clinical Epidemiology, Capital Medical University, Beijing, China.

Received: 17 October 2019 Accepted: 13 December 2019

Published online: 13 January 2020

\section{References}

Alvarado-Esquivela C, Loera-Moncivaisa N, Hernandez-Tinocob J, SanchezAnguianob LF, Hernandez-Madridc G, Rabago-Sancheza E et al (2017) Lack of association between Toxoplasma gondii infection and diabetes mellitus: a matched case-control study in a Mexican population. J Clin Med Res 9:508-511

American Diabetes Association (2010) Diagnosis and classification of diabetes mellitus. Diabetes Care. 31:62-69

Bessman AN, Sapico FL (1992) Infections in the diabetic patient: the role of immune dysfunction and pathogen virulence factors. J Diabetes Complications 6:258-262

Butler AE, Janson J, Bonner-Weir S, Ritzel R, Rizza RA, Butle PC (2013) Beta-cell deficit and increased beta-cell apoptosis in humans with type 2 diabetes. Diabetes 52:102-110

Carter CJ (2013) Toxoplasmosis and polygenic disease susceptibility genes: extensive Toxoplasma gondii host/pathogen interactome enrichment in nine psychiatric or neurological disorders. J Path. 965046

Centers for Disease Control and Prevention (2015) Parasites - toxoplasmosis (toxoplasma infection). CDC, Atlanta http://www.cdc.gov/parasites/ toxoplasmosis/epi.html. Accessed 18 July 2019

Centers for Disease Control and Prevention (2017) Neglected parasitic infections (NPIs) in the United States. CDC, Atlanta http://www.cdc.gov/parasites/npi/ index.html. Accessed 18 July 2019

Cong W, Liu GH, Meng QF, Dong W, Qin SY, Zhang FK et al (2015) Toxoplasma gondii infection in cancer patients: prevalence risk factors genotypes and association with clinical diagnosis. Cancer Lett. 259:307-313

Del Grande C, Galli L, Schiavi E, Dell'Osso L, Brusch F (2017) Toxoplasma gondii a trigger of bipolar disorder? Pathogens 6. https://doi.org/10.3390/ pathogens6010003

DerSimonian R, Laird N (1986) Meta-analysis in clinical trials. Control Clin Trials. 7: 177-188

Donath MY, Ehses JA, Maedler K, Schumann DM, Ellingsgaard H, Eppler R et al (2005) Mechanisms of $\beta$-cell death in type 2 diabetes. Diabetes. 54:S108 
Dupey JP (2008) The history of Toxoplasma gondii - the first 100 years. J Eukaryot Microbiol. 55:467-475

Eckel RH, Kahn SE, Ferrannini E, Goldfine AB, Nathan DM, Schwartz MW et al (2011) Obesity and type 2 diabetes: what can be unified and what needs to be individualized? Diabetes Care. 34:1424-1430

Flegr J (2013) Influence of latent Toxoplasma infection on human personality physiology and morphology: pros and cons of the Toxoplasma-human model in studying the manipulation hypothesis. J Exp Biol. 216:127-133

Gokce C, Yazar S, Bayram F, Gundogan K, Yaman O, Sahin I (2008) AntiToxoplasma gondii antibodies in type 2 diabetes. Nat Med J India. 21:51

Gonzalez-Perez A, Schlienger RG, Rodríguez LA (2010) Acute pancreatitis in association with type 2 diabetes and antidiabetic drugs a population-based cohort study. Diabetes Care 33:2580-2585

Halonen SK, Weiss LM (2013) Toxoplasmosis. In: Garcia HH, Tanowitz HB, Del Brutto $\mathrm{OH}$ (eds) Handbook of clinical neurology Vol. 114 (3rd series) Neuroparasitology and Tropical Neurology. Elsevier B.V, Amsterdam, Netherlands, pp 125-145

Han Y, Nie L, Ye X, Zhou Z, Huang S, Zeng C et al (2018) The association between Toxoplasma gondii infection and hypertensive disorders in T2DM patients: a case-control study in the Han Chinese population. Parasit Res. 117:689-695

Hassanain MA, El-Fadaly HA, Hassanain NA (2014) Toxoplasma gondii parasite load elevation in diabetic rats as latent opportunistic character. Ann Trop Med Pub Health 7:110-115

Hemida MH, Shahat SAR, Bayoumy AMS, Mohammad AD (2017) Prevalence of toxoplasmosis among diabetic patients. Euro J Pharm Med Res. 4:137-140

Henriques SA, Brett R, Alexander J, Pratt J, Roberts CW (2009) Neuropsychiatric disease and Toxoplasma gondii infection. Neuroimmunomod. 16:122-113

International Diabetes Federation (2017) IDF Diabetes Atlas, 8th edition. IFD, Brussels, Belgium

Kankova S, Sulc J, Flegr J (2010) Increased pregnancy weight gain in women with latent toxoplasmosis and RhD-positivity protection against this effect. Parasitology. 137:1773-1779

Khalili M, Mahami-Oskouei M, Shahbazi A, Safaiyan A, Mohammadzadeh-Gheshlaghi N, Mahami-Oskouei L (2018) Correlation between serum levels of anti-Toxoplasma gondii antibodies and the risk of diabetes. Iran J of Parasit. 13:637-642

Li Y, Xin H, Zhang X, Wei C, Duan Y, Wang H, et al. Toxoplasma gondii infection in diabetes mellitus patients in China: seroprevalence, risk factors, and casecontrol studies. BioMed Res Int. 2018; https://doi.org/10.1155/2018/4723739

Majidiani H, Dalvand S, Daryani A, Galvan-Ramirez ML, Foroutan-Rad M (2016) Is chronic toxoplasmosis a risk factor for diabetes mellitus? A systematic review and meta-analysis of case-control studies. Braz J Infec Dis. 20:605-609

Mantel N, Haenszel W (1959) Statistical aspects of the analysis of data from retrospective studies of disease. J Nat Cancer Instit. 22:719-748

Modrek MJ, Saravani R, Mousavi M, Khorashad AS, Piri M (2015a) Investigation of lgG and IgM antibodies against Toxoplasma gondii among diabetic patients. Int J Infect. 2:e27595

Moher D, Liberati A, Tetzlaff J, Altman DG. The PRISMA Group Preferred Reporting Items for Systematic Reviews and Meta-Analyses: the PRISMA Statement. PLOS Med. 2009; https://doi.org/10.1371/journalpmed1000097.

Molan A, Nosaka K, Hunter M, Wang W (2018) A systemic review and metaanalysis of human case-control studies examining the association between Toxoplasma gondii and type 2 diabetes mellitus. Am J Life Sci Res. 6:106-122

Molan AL, Ismail MH. Study the possible association between toxoplasmosis and diabetes mellitus in Iraq. WJPPS. 2017; https://doi.org/10.20959/wjpps20173-8721.

Nazligulm Y, Sabuncu T, Ozbilge H (2001) Is there a predisposition to intestinal parasitosis in diabetic patients? Diabetes Care. 24:1503-1504

Nihad SF, Hamad SS (2017) Seroprevalence of Toxoplasma gondii infection in diabetic patients at Kirkuk province. IJSBAR. 36:157-165

Oz H (2014) Toxoplasmosis, pancreatitis, obesity, and drug discovery. Pancreat Disord Ther. 4:138

Pappas G, Roussos N, Falagas ME (2009) Toxoplasmosis snapshots: global status of Toxoplasma gondii seroprevalence and implications for pregnancy and congenital toxoplasmosis. Int J Parasitol. 39:1385-1394

Pradhan A (2007) Obesity metabolic syndrome and type 2 diabetes: inflammatory basis of glucose metabolic disorders. Nutr Rev. 65:152-156

Prandota J. T. gondii infection acquired during pregnancy and/or after birth may be responsible for development of both type 1 and 2 diabetes mellitus. J Diabetes Metab. 2013; https://doi.org/10.4172/2155-6156.1000241.

Prandovszky E, Gaskell E, Martin H, Dubey JP, Webster JP, McConkey GA (2011) The neurotropic parasite Toxoplasma gondii increases dopamine metabolism. PLoS One. 6:e23866
Reeves GM, Mazaheri S, Snitker S, Langenberg P, Giegling I, Hartmann A, et al. A positive association between $T$. gondii seropositivity and obesity. Front Public Health. 2013;1: https://doi.org/10.3389/fpubh.2013.00073.

Saheb E (2017) Detection of toxoplasmosis infection in diabetic patients. Diyala J Med. 1:70-74

Saki J, Shafieenia S (2016) Foroutan-Rad M. Seroprevalence of toxoplasmosis in diabetic pregnant women in southwestern of Iran. J Parasit Dis. 40:4586-1589

Shirbazou S, Delpisheh A, Mokhetari R, Tavakoli G (2013) Serologic detection of anti Toxoplasma gondii infection in diabetic patients. Iran Red Crescent Med J. 15:701-703

Siyadatpanah A, Tabatabaie F, Oormazdi H, Meamar AH, Razmjou E, Hadighi R et al (2013) Comparison of anti-toxoplasma lgG and IgM antibodies determined by ELISA method in diabetic and non-diabetic individuals in west Mazandaran province, Iran, 2011-2012. Ann Bio Res. 4:281-285

Waree P (2008) Toxoplasmosis: pathogenesis and immune response. Thammasat Med J. 8:487-496

World Health Organization (2014) Global status report on noncommunicable diseases 2014. WHO, Geneva https://www.who.int/nmh/publications/ncdstatus-report-2014/en/. Accessed 12 July 2019

World Health Organization (2016) Global health estimates: deaths by cause, age, sex and country, 2000-2016. WHO, Geneva https://www.who.int/healthinfo/ global_burden_disease/en/. Accessed 15 July 2019

\section{Publisher's Note}

Springer Nature remains neutral with regard to jurisdictional claims in published maps and institutional affiliations.

\section{Submit your manuscript to a SpringerOpen ${ }^{\circ}$ journal and benefit from:}

- Convenient online submission

- Rigorous peer review

- Open access: articles freely available online

High visibility within the field

- Retaining the copyright to your article

Submit your next manuscript at $\boldsymbol{\nabla}$ springeropen.com 